\title{
Les plaies de la Grande Guerre dans la Berliner IIlustrirte Zeitung, un imaginaire de papier sous la République de Weimar
}

\author{
Bérénice Zunino \\ Université de Bourgogne Franche-Comté - CRIT (EA 3224), chercheuse partenaire de SIRICE - Sorbonne Université
}

Abstract

Auch nach dem Waffenstillstand wird der Erste Weltkrieg in der Berliner Illustrirten Zeitung (BIZ) - einem der ersten illustrierten Massenblätter - weiter thematisiert. Bei der Untersuchung dieses mediatisierten Krieges wird der Frage nachgegangen, was diese Bilderwelt über die fortschrittlichen Erwartungen, Ängste und Hoffnungen in der Weimarer Republik aussagt. Dabei geht hervor, dass die BIZ nach der anfänglichen Bekundung eines gewissen Wohlwollens gegenüber den ehemaligen Feinden zu Beginn des Jahres 1919 bald in einen bitteren Ton umschlägt, als die Friedensbedingungen bekannt gegeben werden. Diese enttäuschten Hoffnungen haben die kulturelle Demobilisierung der $B I Z$ erschwert. Eine Zeit lang werden Themen der Kriegspropaganda weiter behandelt, bevor diese illustrierte Zeitschrift im Laufe der 1920er Jahre zum künstlerischen Ausdrucksort der Trauer um die Opfer des Krieges wird und dabei versucht, die Wunden des Krieges zu heilen. Letztlich zielt die BIZ aber weniger darauf ab, die Erinnerung an den Krieg wach zu halten, als vielmehr als Sprachrohr eines pazifistischen und republikanischen Diskurses zu fungieren.

Après l'armistice, la Première Guerre mondiale continue à être traitée dans la revue illustrée d'actualité générale, politique et culturelle qu'est la Berliner Illustrirte Zeitung (BIZ). Sur la trace de cette médiatisation de la guerre, nous interrogeons ce que ces représentations " disent » des attentes, des peurs et des espoirs des contemporains progressistes de Weimar. II ressort qu'après avoir diffusé une certaine bienveillance à l'égard des anciens ennemis début 1919, la BIZ s'aigrit à la suite de l'officialisation des conditions de paix. Sans doute ces espoirs déçus ont-ils compliqué la démobilisation culturelle de la $B I Z$, qui continue un temps à se faire le relais du discours de guerre, puis tente par l'expression artistique, tout au long des années 1920, de panser les plaies du deuil. Finalement, la BIZ ne cherche pas tant à entretenir la mémoire de la guerre qu'à promouvoir un discours pacifiste et républicain.

After the armistice, World War One remained an important topic in the Berliner Ilustrirte Zeitung - an illustrated magazine devoted to general, political and cultural news. Analyzing its coverage of the war allows us to address what these representations have to say about the expectations, fears and hopes of Weimar progressives at the time. After showing some benevolence towards former enemies at the beginning of 1919 , the $B I Z$ grew more acrimonious when the terms of the peace treaty became official. These disappointed hopes certainly made the cultural demobilization of the $B I Z$ more difficult. The magazine continued to relay the war rhetoric for some time, before trying to tend to the wounds and ease the mourning through artistic means. Eventually, the BIZ did not seek to preserve the memory of the war, but rather to promote a pacifist and republican way of thinking.

\section{Keywords}

illustrierte Presse • Erster Weltkrieg • Weimarer Republik • kollektives Gedächtnis • Pazifismus

presse illustrée $•$ Grande Guerre • République de Weimar • mémoire collective • pacifisme

illustrated press $•$ Great War $•$ Weimar Republic $•$ collective memory $•$ pacifism

L'essor de la presse ne peut être séparé de l'affirmation concomitante des États-nations, au cœur de la modernité médiatique (Kalifa / Régnier / Thérenty / Vaillant $2011: 7,13)$. Si la deuxième génération de presse illustrée, née au début des années 1840 en Angleterre, s'adressait encore à un lectorat principalement bourgeois, l'actualité illustrée devint accessible à un plus large public avec la troisième génération, dans les années 1860-1870. Au même moment s'y multiplièrent les thèmes guerriers, favorisés par la guerre franco-prussienne de 1870-1871. S'y développèrent dès lors des imaginaires nationaux au détriment d'un référent collectif européen (Bacot 2005 : 131, 139, 150). Cette presse illustrée au lectorat élargi contribua ainsi à la construction des identités nationales (Thiesse 2010 : 135-136). 
Fondée en 1891 et parue jusqu'au 29 avril 1945, la Berliner Illustrirte Zeitung $(B I Z)$ a sans aucun doute participé de ce phénomène. Cette revue illustrée d'actualité générale, politique et culturelle éditée par Ullstein à partir de 1894, et dont elle fut l'un des plus gros succès, a pourtant encore relativement peu retenu l'attention des chercheurs puisqu'aucune monographie ne lui a été consacrée à ce jour ${ }^{1}$.

Dans la $B I Z$, la guerre devint un enjeu médiatique à partir de la guerre russo-japonaise, en 1904 (Gervais 2015 : 44), avant de s'affirmer comme l'un des thèmes majeurs du photojournalisme pendant la Grande Guerre. Comme la plupart des magazines en France et en Allemagne, ce qu'on peut décrire comme le premier illustré de masse a largement œuvré à la mobilisation culturelle entre 1914 et 1918 (Beurier 2016 : 114, 191 ; Schmitz 2014). Bien qu'aux accents moins violents que leurs équivalents français, les revues illustrées d'outreRhin, dont la $B I Z$, n'enterrèrent pas la guerre en 1918 : elles continuèrent à véhiculer des représentations guerrières atemporelles après l'armistice et contribuèrent à " fabriquer le sentiment de l'impossible défaite " (Beurier 2016 : 427). Mais alors que, de 1914 à 1918, la BIZ consacrait, selon les années, entre $38 \%$ et $64 \%$ de ses images à des thèmes militaires et guerriers, leur part baissa rapidement après 1919 pour ne pas dépasser 2\% durant les années 1920 (Dussel 2019 : 361). Dès lors, pourquoi s'intéresser à la survivance de la Grande Guerre dans cette revue illustrée sous la République de Weimar?

On sait que Weimar fut « un enfant de la Grande Guerre » (Krumeich 2018 : 11), héritière d'une part de la violence politique cumulée de l'expérience de guerre, de la Révolution de novembre et de sa répression, et d'autre part d'un traité de paix honni par tous les acteurs politiques. S'inscrivant tout comme sa concurrente conservatrice Die Woche (éditée par Scherl) (Dussel 2019 : 29) dans ce consensus, la BIZ, orientée à gauche, réserva elle aussi un traitement privilégié à l'amertume causée par les conditions de paix imposées aux Allemands. Elle s'en est faite d'autant plus ardemment le vecteur qu'en tant que revue illustrée de masse, largement diffusée, elle se savait en adéquation avec ses lecteurs. À raison d'un numéro à 20 pfennigs pièce ou d'un abonnement mensuel à 3 marks, elle était accessible aux classes moyennes autant qu'à un lectorat aux moindres revenus (Dussel 2016 : 31). Ses tirages s'élevaient à un million d'exemplaires dès 1914 avant de baisser à 450000 au plus fort de la crise économique en 1923, puis de se redresser à 1,75 million d'exemplaires fin 1926 et d'atteindre son record de plus de 1,8 million en 1930 (Dussel 2012 : 189).

Dans ce contexte, la $B I Z$ joua un rôle central dans la nouvelle culture de masse de Weimar rendue possible par le nouvel État démocratique et associée à l'émergence d'une société civile moderne (Becker 2018 : 11, 47, 262). La Première Guerre mondiale a effectivement été vue comme un facteur ayant permis la modernisation sociale et culturelle sous Weimar et l'historiographie a montré que la République

On trouvera en bibliographie les ouvrages principaux traitant de la BIZ. Une exposition a également été organisée récemment au Musée historique allemand (Stiftung Deutsches Historisches Museum 2017). allemande n'était pas nécessairement vouée à un échec imputable à l'insuffisance d'un pouvoir politique souvent opposé au rayonnement de la modernité culturelle (Graf 2008 : 371 ; Maubach 2018 ; Reimann 2004 : 35). Canal d'expression d'écrivains - Thomas Mann, Vicki Baum, Leo Perutz, Kurt Tucholsky - et d'artistes - Theo Matejko, Heinrich Zille, Umbo, Martin Munkacsi - la BIZ participa de l'ouverture de la littérature, du théâtre et des arts visuels à cette culture de masse, contribuant à l'intermédialité des nouveaux médias - presse illustrée, radio, cinéma (Becker 2018 : 19). Après l'apparition d'innovations techniques dès les années 1890 - similigravure, presses rotatives, premiers appareils Kodak - la culture de Weimar marqua la professionnalisation croissante du photojournalisme (Vowinckel 2016 : 55). Au prisme de cette modernité culturelle et politique, la présente contribution se propose d'étudier la survivance de la mémoire de la Grande Guerre dans cette revue de masse au moment de sa " sortie de guerre " (Krumeich 2008).

Concomitamment à ses conséquences politiques médiatisées, le conflit reste présent, en creux, sous la forme de certains sujets culturels, artistiques et techniques dans la $B I Z$; il apparaît ou réapparaît au détour d'un article sur la vie quotidienne, l'art du voyage ou les expositions d'après-guerre. En traquant ses traces, ses invariants et ses variations lovées dans cet imaginaire de papier, nous nous proposons d'interroger ce que "disent " ses représentations des attentes, des peurs et des espoirs des contemporains progressistes de Weimar. Pour ce faire, nous avons analysé l'intégralité du contenu de l'année qui suit directement l'armistice ainsi que celles qui marquent les grands anniversaires de la guerre et de la Conférence de la paix - 1924, 1928 et 1929 - plus propices, pensions-nous, aux élans commémoratifs. II ressort qu'après avoir diffusé une certaine bienveillance à l'égard des anciens ennemis, la BIZ s'aigrit à la suite de l'officialisation des conditions de paix. Sans doute ces espoirs déçus ont-ils compliqué la démobilisation culturelle de la $B I Z$, qui continue un temps à se faire le relais du discours de guerre, puis tente par l'expression artistique, tout au long des années 1920, de panser les plaies du deuil. Finalement, la BIZ ne cherche pas tant à entretenir la mémoire de la guerre qu'à promouvoir un discours pacifiste et républicain.

\section{ESPOIRS DÉÇUS DE 1919 : FEMMES ET ENFANTS, SYMBOLES DU MARTYR DE L'ALLEMAGNE}

Au sortir de la guerre la $B I Z$, comme toute la presse, traverse une crise morale et économique notoire. Sa contribution à la mobilisation culturelle a provoqué une perte de crédit auprès du public (Weise 1997 : 81). Le magazine subit de plus les conséquences de l'inflation. Dès 1917, il est contraint d'augmenter son prix de vente jusqu'au pic inflationniste de 1923. De 25 pfennigs au 10 août 1919, le prix du numéro passa à 80 marks fin 1922 pour atteindre 50 milliards de marks (!) lors de I'hyperinflation de novembre $1923 \ldots$ avant de retrouver 
son prix standard de 20 pfennigs le 25 novembre (Dussel 2019 : 43, 73-74).

Jusqu'en 1919, la guerre figure au centre de l'actualité politique et sociale. Du 17 novembre au 29 décembre 1918, pas moins de six Une abordent la démobilisation. L'année suivante, douze Une sont en lien avec la guerre, dont trois sont consacrées aux revendications de la libération des prisonniers et six aux conditions de paix. Après le soulagement suscité par le retour des troupes, malgré la défaite, la $B I Z$ s'attache, début 1919 , à mettre en avant les difficultés et souffrances communes des pays belligérants, vaincus comme vainqueurs. Mais cette ouverture européenne est de courte durée. Face à la poursuite du blocus et aux conditions de paix perçues comme injustes, un changement de ton s'amorce au printemps. Soulagement et espoirs font place à la désillusion. Au cours de ces différentes phases, femmes et enfants - victimes innocentes de la guerre - incarnent le « martyr » de la population allemande.

La BIZ consacre une part importante de ses numéros de novembre et décembre 1918 à l'actualité révolutionnaire à Berlin, à la mise en place du gouvernement provisoire et au retour des troupes. Ce dernier thème est aussi bien traité sous la forme de clichés de leur arrivée " triomphale » que par des images plus ambivalentes oscillant entre soulagement et tristesse. Dans tous les cas est véhiculé un discours compassionnel stéréotypé qui s'inscrit dans la continuité des représentations du temps de guerre (Winter 2008 : 144-149) et relativise la défaite. Des dessins et reportages photographiques conformes aux mises en scène de l'accueil " triomphal » des troupes dans les villes allemandes sont visibles dans presque tous les numéros : on y voit des soldats disciplinés et rassurants, des enfants curieux et des foules en liesse. Le photoreportage « Le sort du Feldgrau. À propos du retour de nos troupes » est représentatif de la manière dont la défaite est alors transmutée en victoire ; dans la continuité du discours de guerre, l'Allemagne y est décrite comme encerclée par une horde d'ennemis face auxquels - est-il affirmé rétrospectivement - elle n'aurait pu s'imposer. S'ensuit un discours compassionnel en hommage à la ténacité des soldats :

Für den Sturmangriff ,kräftigte' er sich mit Marmelade und Hering, um dann in den erstürmten Linien den unvorstellbarsten Reichtum der Verpflegung und Ausrüstung bei seinen Gegnern zu sehen. Niemand half inm, nicht seine Führer, die gleichermaßen die feindlichen Mittel unterschätzten, wie sie den Grad seiner Unerschöpflichkeit blind und taub überschätzten [...]. Und im Rücken hatte er die Heimat seiner Kinder, seiner Frau, deren Hunger inn niederdrückte (BIZ 01/12/1918 : 378-379).

La critique publique des autorités militaires, rendue possible par la levée de la censure et le rétablissement de la liberté de la presse le 12 novembre 1918 (Kübler 2007 : 56), témoigne d'un relâchement et d'une libération de la parole qui auraient été impensables en guerre. Ambivalentes, les souffrances de l'arrière rappellent les accusations colportées par la Légende du coup de poignard dans le dos et visent simultanément à susciter autant l'empathie envers les civils - victimes du blocus - que l'admiration à l'égard des soldats. Après avoir relaté le retrait ordonné des troupes, le reportage photographique se concentre sur le retour à la vie civile : par un processus d'identification, les lecteurs suivent alors un individu ayant quitté l'anonymat de son régiment pour retrouver sa famille ; la photo centrale, dont la forme ovale « en médaillon » revêt un caractère intimiste, le présente en père de famille serrant sa fillette dans les bras ${ }^{2}$. L'article s'achève sur un éloge du combattant allemand, le Feldgrau (en référence à son uniforme vert-de-gris), qui a " battu tous les records, le record des victoires, le record de la ténacité, le record du moral et celui de l'abnégation » (BIZ 01/12/1918 : 379). Finalement, ces invariants narratifs - l'héroïsation des soldats conjuguée aux figures de la femme et de l'enfant concourent à brosser le portrait d'un peuple allemand méritant et invaincu. Tout concorde à véhiculer l'idée du « contrat rempli » (Jardin 2005 : 445) - et contribue au déni de la défaite. À l'aide d'un imaginaire familier, la $B I Z$ met aussi en image la stupeur causée par l'annonce de la défaite, et laisse entrevoir la détresse de la population. Un dessin intitulé "Les retrouvailles » en Une du numéro du 1 ${ }^{\text {er }}$ décembre 1918 attire le regard par son ambivalence ${ }^{3}$. Un couple s'étreint, le soldat campé sur ses deux jambes, son allure rassurante et protectrice accentuée par la vue en contre-plongée ; on imagine facilement la femme en robe longue accourir, l'instant d'avant, dans les bras de son bien-aimé. Son visage est lové contre son épaule - pleure-t-elle ? Ici, pas de signe d'allégresse. Le calme d'un paysage alpin propice au recueillement se substitue à la foule en liesse de la métropole. Les codes conventionnels des couples d'amoureux sur les cartes postales patriotiques se voient remobilisés pour exprimer à la fois soulagement et détresse causée par les souffrances psychologiques endurées au cours de longues années d'attente. On est loin d'un triomphalisme à toute épreuve.

La même sobriété ressort des reportages dédiés aux préoccupations économiques et sociales des pays voisins. De la mi-décembre 1918 à début mai 1919, la BIZ fait preuve d'un esprit d'ouverture à l'égard des puissances anciennement ennemies en rendant compte du retour des troupes à Paris et à Londres, de la politique de réintégration des mutilés sur le marché du travail anglais et des difficultés matérielles auxquelles sont confrontés les Parisiens pour se nourrir et se loger (BIZ 14/12/1918 : 394-395 ; BIZ 26/01/1919 : 28 ; BIZ, $11 / 05 / 1919$ : 154). Ces reportages restent, certes, ambivalents : teintés d'une certaine méfiance - ou d'une Schadenfreude ? - ils s'avèrent utiles pour relativiser la détresse de la population allemande et minimiser, de manière détournée, la gravité de la défaite. Ils révèlent néanmoins un intérêt pour l'autre et une volonté de rapprochement entre les peuples. Ce journalisme à dimension européenne tend en fin de compte à créer une " communauté de souffrances " qui dépasserait les frontières nationales et les alliances d'hier.

2 La page du reportage photographique est consultable au lien suivant : https://argonnaute.parisnanterre.fr/ark:/14707/a011446652157f2Qhdo/0115a5d917 La collection de la BIZ détenue par La Contemporaine est entièrement numérisée : https://argonnaute. parisnanterre.fr/ark:/14707/a011446477836Hq49gs/from/a011406131043hqToci

3 Cette Une est consultable au lien suivant : https://argonnaute.parisnanterre.fr/ ark:/14707/a011446652157f2Qhdo/d5335acedb 
Après la remise des conditions de paix à la délégation allemande début mai, le ton de la $B I Z$ connaît toutefois un revirement. Choqués par la prolongation du blocus après l'armistice, les Allemands accueillent avec indignation les conditions de paix imposées par les vainqueurs et se sentent trahis par un traité qui leur fait porter la responsabilité principale de la guerre. Deux Une et plusieurs photoreportages rendent compte des manifestations de protestation contre le « diktat » (BIZ 25/05/1919 : 1, 179 ; BIZ 01/06/1919 : 1, 190). L'avenir est trop incertain et les plaies, trop vives pour faire place à un rapprochement entre vainqueurs et vaincus. Le relatif esprit d'ouverture et l'optimisme nourri par l'espoir d'une paix de compromis sur la base des 14 points de Wilson - surnommé quelques années plus tard dans un article "Président Wilson, l'impuissant "(BIZ 13/01/1924 : 22) cèdent la place à un recentrage des reportages sur les souffrances de la population allemande. Cette situation réactive l'amertume suscitée par la détention prolongée des prisonniers allemands - alors que la libération sans délai des prisonniers alliés avait été l'une des conditions non-négociables de l'armistice, sans réciprocité (Grevelhörster 2005 : 134). Ce leitmotiv va crescendo : la $B I Z$ le relaie dès février 1919 (BIZ 02/05/1919 : 35 ; BIZ 09/02/1919 : 45) avant d'y consacrer trois Une entre juin et décembre. Celle du 21 décembre 1919 convoque une figure féminine : une femme, la tête entre les mains, étouffe des sanglots de désespoir ${ }^{4}$; à ses mains rugueuses, usées par le travail, on suppose aisément qu'il s'agit d'une mère meurtrie par l'absence prolongée de son fils ; le titre est éloquent : « Peuple en détresse. Il est toujours détenu en France ! » (BIZ 21/12/1919 : 1). Ici encore, la figure maternelle est mobilisée pour symboliser l'affliction et la détresse de la population allemande après d'immenses sacrifices. Femmes et enfants sont également réquisitionnés pour dénoncer les conditions de vie, aggravées par la prolongation du blocus après l'armistice, jusqu'à la signature du traité de paix. Dans un photoreportage consacré à la pénurie alimentaire $^{5}$, le blocus - responsable de la majorité des 760000 victimes civiles mortes de faim ou de sous-alimentation depuis 1914, surtout de jeunes enfants - est comparé au massacre des Innocents :

Ueber den bethlehemitischen Kindermord, der vielleicht hundert Knaben das Leben gekostet hat, haben sich die Menschen zweitausend Jahre entsetzt. Weiß die ganze Welt, dass die Hunger-Blockade in Deutschland tausend und abertausend Kinder dahingerafft hat und noch immer täglich hunderte tötet? (BIZ 01/06/1919 : 192)

Cette surenchère dans l'horreur s'accompagne de la " photo-choc " d'un enfant sous-alimenté au corps décharné. Son regard épuisé dévisage les lecteurs-spectateurs. Le petit corps est maintenu debout par une infirmière dont les traits harassés ne laissent aucun doute sur l'issue fatale de la situation. Ce cliché, au centre du photoreportage, se détache de

4 Voir : https://argonnaute.parisnanterre.fr/ark:/14707/a0114467151508fcDWO/a504023737 5 Consultable au lien suivant : https://argonnaute.parisnanterre.fr/ark:/14707/ a0114467151508fcDWO/2f2287c48d l'arrière-plan formé par deux photographies d'un marché couvert et d'abattoirs vides - les légendes renforcent leur lecture selon un schéma interprétatif causes-conséquences : "Les conséquences du blocus de la faim en Allemagne : la grande halle d'un marché couvert berlinois aux étals vides ", suivie des « Conséquences atroces du blocus : un garçonnet de six ans rendu tuberculeux et impotent par la sous-nutrition » (BIZ 01/06/1919: 192).

Même après la levée du blocus, les figures enfantine et maternelle continuent à incarner le martyr de la population allemande. Dans une double-page consacrée aux difficultés matérielles - hyperinflation, pénurie de vêtements, de charbon, froid, faim - et à l'inquiétude causée par « les nouvelles quotidiennes alarmantes sur ce que l'Entente trame de nouveau et d'horrible contre l'État vaincu », le portrait d'un jeune garçon aux joues creusées par la faim («Peuple en détresse : Le visage meurtri par la faim d'un enfant de la métropole aujourd'hui ») et la scène d'enfants attablés dont la mère essaie d'allumer le poêle avec du bois et du papier («Maman, il fait si froid ! ») érigent l'Allemagne en victime, en proie aux attaques des vainqueurs en train d'« anéantir un peuple qui voulait sincèrement la paix » (BIZ 21/12/1919: 530-531). Lorsque les espoirs d'une paix de compromis respectant le droit des peuples à disposer d'eux-mêmes s'avèrent illusoires, ces figures universelles de l'innocence incarnent le sort de la nation.

Au sortir de la guerre, c'est donc l'amertume qui prédomine. Peut-on alors supposer que la dureté des conditions de paix a compliqué la démobilisation culturelle à l'œuvre dans la BIZ ?

\section{CICATRICES ARTISTIQUES ET EXPRESSION DU DEUIL}

Avant même que les conditions de paix ne soient rendues publiques, la $B I Z$ se caractérise par son attrait pour les aspects culturels de la guerre passée. La propagande ennemie par l'image, vilipendée durant le conflit et qui resta une préoccupation après 1918 (Avenarius 1918), fait l'objet de vives critiques et offre aux journalistes un moyen efficace d'ériger l'Allemagne en victime d'un complot allié (BIZ 11/05/1919: 158-159). C'est toutefois en raison du « diktat » que le ton se durcit. Dès lors, l'indignation s'étend au champ culturel. L'article « La spoliation du retable de Gand » (BIZ 25/05/1919: 178), par exemple, s'insurge contre la clause du Traité de Versailles imposant la restitution à la Belgique des panneaux latéraux de L'Agneau mystique et de La Dernière Cène du retable du Saint Sacrement en compensation des dommages de guerre. Par un procédé d'inversion des rôles - les troupes allemandes s'étaient livrées à de nombreux pillages et destructions en Belgique et dans le Nord de la France durant le conflit (Kott 2006) - l'Allemagne s'érige ici en victime « spoliée " par les vainqueurs.

La vision doloriste n'est pas le seul procédé à l'œuvre. À l'occasion d'un passage en revue des expositions proposées dans la capitale berlinoise est reproduit un tableau en hommage au « héros de Tannenberg » : Hindenburg am 
14. August 1914 in Marienburg (BIZ 08/06/1919 : 202-203) ${ }^{6}$. Peut-on supposer que la résurgence de cette figure charismatique (Pyta 2007 : 95), au moment où l'Allemagne se sent humiliée par le traité de paix, n'est pas fortuite ? De fait, la $B I Z$ aime à soigner l'image de la nation et de sa capitale. Cette fierté patriotique aux relents de culture de guerre atteint son paroxysme dans un article prônant, à l'occasion des fêtes de Noël, la qualité de l'industrie du jouet allemande par rapport à sa concurrente anglaise, avec le témoignage d'une lectrice du Times à l'appui (BIZ 28/12/1919 : 542-543).

Ces reliquats de la culture de guerre ne subsistent toutefois pas longtemps. Parmi les numéros de 1924, 1928 et 1929 , un seul article se voit parasité par la langue de bois de 1914-1918. Consacré à l'apport civilisationnel du progrès technique, il répond à une tribune d'Alexandre Moszkowski ; l'auteur de Die Inseln der Weisheit y avait déploré le potentiel destructeur des armes modernes et imputé le déclenchement de la guerre aux nouveaux moyens de communication, qui, par leur rythme effréné, auraient précipité les contemporains dans un conflit généralisé (BIZ 08/01/1928 : 57-59). Dans une démarche apparentée à certains courants de la révolution conservatrice, son contempteur Walter von Dyck réactive les topoï afférents au narratif d'une nation encerclée contrainte à mener une guerre de défense :

Die Schuld aber am Weltkriege? Trifft sie nicht vielmehr die Drahtzieher der feindlichen Politik als die ,Hexendrähte' der Telegraphie und Telephonie, die doch nur die Auslösung vermittelt haben einer von lange her aus Machtfragen der Politik und des Geldes erwachsenen Spannung, die in einer von Neid, Missgunst und Hass gegen das emporblühende Deutsche Reich erfüllten, gewitterschwülen Atmosphäre sich gebildet hatte? Und um beim Krieg zu bleiben, war es nicht die Technik, die uns, umringt von Feinden, lange Jahre hindurch den aufgezwungenen Kampf bestehen ließ, draußen mit den besten Angriffs- und Verteidigungswaffen, die die Technik uns bieten konnte, in der Heimat durch die von Naturwissenschaft und Technik dargebotenen Hilfsmittel, ein von der Außenwelt völlig abgeschnittenes Volk zu nähren, zu kleiden und gesund zu erhalten [...]! (BIZ 15/01/1928: 97)

Les arguments avancés par l'auteur s'inscrivent dans le long débat des causes et de la responsabilité du conflit, et se démarquent par leur virulence. Hormis ces exceptions, les ressentiments disparaissent rapidement de la $B I Z$ pour faire place à un traitement « technique » de la guerre, l'un des thèmes de prédilection de la revue illustrée. Très tôt, la BIZ cultive le goût pour les innovations du temps de guerre, par des récits dignes du roman d'anticipation, prédisant par exemple l'usage des chars à des fins agricoles (!) (BIZ 27/04/1919 : 131). C'est dire si cet illustré fut aussi un canal de diffusion de la littérature de science-fiction émergente.

Plus que l'animosité caractéristique de l'ancienne culture de guerre, la mémoire du conflit trouve surtout son expression dans l'art et le deuil. Malgré ses élans d'optimisme et ses images de

6 Une variation du célèbre tableau d'Arthur Kampf, Volksopfer 1813, rebaptisée ici Pro Patria, y est aussi reproduite. II était régulièrement repris dans les cartes postales et les livres pour enfants entre 1914 et 1918. (Zunino 2019 : 91). bonheur familial, la $B I Z$ est habitée, tout au long de la décennie, par un culte artistique du souvenir des morts de la Grande Guerre. CEuvres d'art dédiées aux victimes, photographies de monuments aux morts tout juste inaugurés, dessins de mères endeuillées, hommage à un fils perdu au détour d'un article les lecteurs sont pudiquement confrontés, au fil des pages et des années, aux souvenirs lancinants de la guerre. Ainsi le névrosé de guerre côtoie-t-il, dans un reportage sur les facettes les plus sombres de la métropole digne d'un tableau d'Otto Dix ou de Hans Baluschek, le mendiant, l'ouvrier, la mère de famille des quartiers ouvriers, l'enfant des rues (BIZ 14/09/1924: $1085-1087)$... La mise en image des mères inconsolables illustre le poids du deuil : en témoignent la reproduction de L'Ange volant d'Ernst Barlach (BIZ 05/02/1928 : 203) ou le dessin d'une vieille dame bossue et hagarde, tenant entre les mains le portrait de son fils tombé au front ; le balancier de l'horloge qui dépasse du bord gauche de l'image ne cesse de sonner les heures, celles de son attente angoissée, puis de son chagrin, qui ne prendra fin qu'à sa propre mort (BIZ, 24/11/1929 : 2111). Un autre exemple en date de 1928 révèle à quel point les disparus hantent les contemporains, à peine dix ans après l'armistice ; ce reportage photographique consacré au tourisme de mémoire étonne par le caractère novateur de sa mise en page - les clichés des forts de Vaux et de Douaumont, de la tranchée des baïonnettes et de l'ossuaire de Douaumont (achevé en 1932) sont mis en valeur par un encadré noir inhabituel (BIZ, 15/07/1928 : 1252-1253). Le journaliste voit d'un œil sceptique l'essor du tourisme de champ de bataille près de Verdun. II insiste sur la sacralité du lieu, déshonoré selon lui par un tourisme de mémoire irrévérencieux, voyeuriste et vénal :

Verdun selbst macht heute nach zwölf Jahren den Eindruck, als wäre es erst vor ein paar Wochen beschossen worden. In der Nähe der Umwandlung überall ausgebrannte und zerschossene Häuser, verrostete Wellblech-Buden, Trümmerhaufen; und unvermittelt zwischen ihnen grelle Neubauten oder Kioske für Ansichtskarten und Andenken (BIZ 15/07/1928 : 1251).

Animé par une méfiance à l'égard d'un possible chauvinisme français, l'auteur poursuit son reportage d'un ton désapprobateur. II y oppose la respectabilité d'une visite solitaire et silencieuse, rendue nécessaire par le deuil et le besoin de recueillement, à l'indécence désinvolte et " profanatrice » des masses mues par la curiosité et la recherche du sensationnel :

Wie wirkt die Führung durch die Schlachtfelder überhaupt auf einen unvoreingenommenen Besucher? Wirkt sie wie eine große Geste des Kriegs gegen den Krieg? Oder wirkt sie aufreizend und chauvinistisch? - Ich habe all diese Stätten einmal allein an einem regnerischen Vormittag besucht. Eine versöhnliche Ruhe lag über dem zerfurchten Boden. Die Silhouette einer Ruine, eines Baumstumpfes, ein verrosteter Drahtverhau - das alles redete die Sprache des Friedens. Im Trubel des darauffolgenden Sonntags jedoch war der Eindruck völlig entgegengesetzt! Eine ganze Reihe von Faktoren ist daran schuld; am meisten aber sind es die Besucher selbst. [...] Es 
sind die Ausflügler, die um die Mittagszeit wie auf Kommando allenthalben ihr Picknick entfalten oder abkochen. Es sind die Durstigen, die sich an der Trinkstube vor dem Ossuaire um Erfrischungen anstellen; die Sammler, die nach Helmen und Granatsplittern fahnden; die Kinder, die in den Laufgräben und auf den Forts Verstecken spielen - es ist der ganze würdelose Vertrieb, der eine Entweihung des Bodens darstellt, von dem jeder Fußbreit mit Blut getränkt ist.

Es gibt auch andere Besucher. Angehörige verschiedener $\mathrm{Na}$ tionen, die ihre Reise hierher vielleicht mit finanziellen Opfern erkauft haben und ihren Vätern und Söhnen einen letzten Besuch abstatten wollen. Sie werden es doppelt peinlich empfinden, dass sich vor Verdun allsonntäglich dieser geschmacklose Schaubetrieb abspielt (BIZ 15/07/1928 : 1254-1255).

Au-delà de l'indignation probablement réelle qui trouve ici son expression, ce ton moralisateur tranche avec la conception actuelle des lieux de mémoire du $X X^{e}$ siècle, mais il révèle aussi la vivacité des plaies de la Grande Guerre dans la société de Weimar. Malgré la méfiance qu'il colporte vis-à-vis des Français, cet article appelle aussi une paix durable de ses vœux. Au cours des années 1920, la BIZ contribua à diffuser un discours en faveur de la consolidation de la paix et de la République.

\section{La BIZ, PACIFISTE MODÉRÉE ET RÉPUBLICAINE}

Sur fond de mémoire clivée du conflit et de sempiternels débats autour des réparations, la $B I Z$ se fait le relais des voix pacifistes qui s'élèvent en Allemagne durant les premières années de la République, jusqu'en 1924-1925 (LütgemeierDavin 1981). Même après que ne l'emporte la mémoire nationale-conservatrice de la guerre, l'illustré conserve sa ligne éditoriale en se préoccupant des dangers d'un possible affrontement futur. Les souffrances de 1914-1918 font place progressivement à l'idée de guerre impossible, et les commémorations du calendrier républicain l'emportent sur celles de la Grande Guerre.

Dix ans après le début des hostilités et dans un contexte de frictions dues à l'occupation de la Ruhr, la $B I Z$ s'inquiète ainsi d'un nouveau risque de guerre en Europe et déplore la « prééminence de la France » dont la sphère d'influence en Europe de l'Est pourrait conduire de nouveau à un " encerclement de l'Allemagne » (BIZ 13/01/1924 : 23). Favorable à l'arbitrage international des conflits par le droit, le magazine revendique l'entrée de l'Allemagne dans la Société des nations et diffuse les idées du pacifiste modéré Ludwig Quidde ainsi que les appels pan-européens de Richard Coudenhove-Kalergi (BIZ 19/10/1924 : 1 ; BIZ 28/12/1924 : 1565-1567). Les arguments pacifistes avancés par la $B I Z$ se fondent sur la technicisation de la guerre : le potentiel de destruction des armes modernes et leurs conséquences atroces sur les civils auraient rendu tout conflit futur impossible et absurde ; exposés scientifiques et témoignages de responsables militaires appuient ce diagnostic (BIZ 19/10/1924 : 1218-1219). Comme dans le magazine français $V U$ - dont la création par Lucien Vogel en 1928 a été inspirée par la BIZ et l'Arbeiter-IIlustrierte-Zeitung (Feyel 2001 : 33 ; Frizot 2006 : 158-159)ainsi que dans nombre de productions artistiques et littéraires, le masque à gaz devient le symbole repoussoir de la guerre moderne (Sloterdijk 2002 : 12). Dans un article au titre éloquent, « Le spectre mondial de la guerre des gaz : la guerre s'autodétruit », le prix Nobel de chimie 1909 met en avant les ravages causés par cette arme chimique pour réclamer la fin définitive de la guerre et plaider en faveur d'une « entente pacifique » (BIZ 08/09/1929: 1574) entre les peuples :

Die Verwendung von Giftgasen im Weltkriege hat blitzartig die fürchterlichen Waffen erkennen lassen, welche die moderne Technik den Kriegführenden in die Hand geben könnte, und wer die technischen Möglichkeiten etwa auf biologischem Gebiet ermisst, der erkennt, dass die plötzliche Vernichtung von Hunderttausenden, ja Millionen Menschenleben im Bereich des Ausführbaren liegt. So hat im zwanzigsten Jahrhundert der Krieg sich selbst unmöglich gemacht (BIZ 08/09/1929 : 1574).

Parmi les images illustrant cette contribution, un dessin visionnaire de Theo Matejko ${ }^{7}$ représente des civils - dont une femme et un enfant au premier-plan - gisant sur un trottoir, victimes d'une attaque aux gaz (BIZ 08/09/1929 : 1573). Le commentaire d'une autre photographie montrant un groupe de jeunes femmes harnachées de masques à gaz (BIZ, 08/09/1929 : 1574) se fait encore plus alarmant :

Nutzlose Bemühungen. Aufmarsch von jungen Mädchen des holländischen Roten Kreuzes, das für die Ausstattung der Bevölkerung mit Gasmasken wirbt. Nach dem Urteil von Sachverständigen bieten schon beim gegenwärtigen Stand der Giftgastechnik weder Gasmasken noch Unterstände sicheren Schutz (BIZ 08/09/1929 : 1574).

Conjuguée à sa « scientificité » affichée, ce reportage ne cesse de recourir à la figure universelle de la femme - jeune pour dénoncer les horreurs de la guerre. En fin de compte, un glissement s'est effectué des images de la Grande Guerre vers celles d'une guerre imaginée et à venir conforme aux craintes des contemporains.

De surcroît, la faible médiatisation des commémorations du premier conflit mondial confirme le relatif effacement de celui-ci, non sans lien avec sa mémoire clivée. Devant l'échec du gouvernement à rassembler les Allemands autour des morts de 1914-1918 (Weinrich 2017 : 110), la BIZ a privilégié le calendrier républicain à la mémoire de la guerre. Dès novembre 1918, la revue accorde une attention particulière à la mise en place de la jeune République parlementaire : actualité politique du gouvernement provisoire, publication régulière de portraits des députés - hommes comme femmes ( $B I Z$ 09/02/1919 : 48) - érigés en une sorte de panthéon républicain, appels à voter à chaque élection : la $B I Z$ fait

7 À la même période, cet illustrateur contribua pourtant de manière virulente à la propagande nationaliste (contre l'occupation de la Ruhr notamment). II mit par la suite son art au service de la presse nazie. 
montre de son soutien à la République. A contrario, seuls deux événements, inscrits aujourd'hui encore dans la mémoire allemande du conflit, sont commémorés en images : le dixième anniversaire de la bataille de Tannenberg, à l'occasion de l'inauguration du monument en Prusse-Orientale (BIZ 14/09/1924 : 1087), et celui de la signature du Traité de Versailles - qui donne lieu à une manifestation de protestation à Cologne (BIZ 14/07/1929 : 1226). Aucune trace des commémorations du début du conflit ni de l'armistice (comment, du reste, commémorer une défaite ?). Conformément à la position du SPD depuis l'échec des commémorations du 3 août 1924, le magazine préfère célébrer la date d'anniversaire de la signature de la Constitution (Verfassungstag) dans des articles solennels et élogieux (BIZ 26/08/1928: 1460-1461 ; BIZ 11/08/1929 : 1412-1413). Même lorsqu'il est furtivement question du défilé de l'organisation paramilitaire républicaine Reichsbanner Schwarz-Rot-Gold sous la Porte de Brandebourg en souvenir des morts de la guerre, la primauté est donnée à la fête nationale et républicaine du 11 août ; en témoignent le titre de l'article, "La célébration des dix ans de la Constitution à Berlin » et les images du reportage photographique, dont une seule montre les slogans concurrents brandis par la Reichsbanner: "À tous les morts de la guerre mondiale » et "Aux victimes de la République et du travail » (BIZ 25/08/1929: 1490-1491). Alors qu'aucun récit fédérateur autour de l'idée républicaine ne put s'implanter sous Weimar (Julien 2009 : 52), la BIZ tenta d'œuvrer à la création d'un mythe fondateur positif de la République.

À la lecture d'un article paru le 11 novembre 1928, intitulé « Dix ans déjà ! Du 9 novembre 1918 au 9 novembre 1928 », on perçoit toutefois la difficulté de cette tâche. Là encore, c'est le renouveau démocratique qui est commémoré, et non l'armistice. Le journaliste fait même montre d'un optimisme certain en affirmant qu'Hindenburg, depuis son élection en 1925 , s'est révélé être un « garant loyal de la Constitution ». Malgré tout, les ravages économiques de l'après-guerre restent au premier plan du reportage : trois des cinq photographies rappellent la sous-nutrition des écoliers en 1919 et l'hyperinflation de 1922-1923 (BIZ 11/11/1928 : 1956). Produit de son temps, la $B I Z$ n'a donc pas été épargnée par les difficultés à légitimer la première démocratie allemande ni par l'impossibilité d'instaurer une mémoire républicanisée de la Grande Guerre. Au cours des années 1920, elle s'est pourtant fait le vecteur d'un discours pacifiste et républicain.

Pour conclure, il ressort que la première revue illustrée de masse, au sortir de la guerre, a médiatisé les événements traumatiques qui marquent aujourd'hui encore la mémoire allemande de cette époque : l'invasion de la Prusse-Orientale, les souffrances des civils, les désillusions suscitées par le traité de paix, l'hyperinflation - ces plaies qui trouvèrent leur expression dans l'art et dans les figures universelles de la femme et de l'enfant. La Grande Guerre n'y représentait toutefois pas une thématique prépondérante, si bien que l'on peut parler d'une démobilisation culturelle partielle de cette revue illustrée. Malgré ces cicatrices, la $B I Z$ diffusa un imaginaire de papier progressiste, artistique, pacifiste et républicain, au cœur d'une modernité culturelle et politique. Elle tenta d'insuffler à ses lecteurs un élan d'optimisme et une confiance en l'avenir, élargissant par ses choix éditoriaux le champ des possibles sous Weimar.

\section{Bibliographie}

Avenarius, Ferdinand (1918), Das Bild als Narr. Die Karikatur in der Völkerverhetzung, was sie aussagt - und was sie verrät, München, Callwey.

Bacot, Jean-Pierre (2005), La presse illustrée au XIX siècle. Une histoire oubliée, Limoges, Presses universitaires de Limoges.

Becker, Sabina (2018), Experiment Weimar. Eine Kulturgeschichte Deutschlands 1918-1933, Darmstadt, WBG Academic.

Beurier, Joëlle (2016), Photographier la Grande Guerre, Rennes, Presses universitaires de Rennes.

Dussel, Konrad (2012), Pressebilder in der Weimarer Republik: Entgrenzung der Information, Berlin, LIT.

Dussel, Konrad (2016), «Wie teuer war ein Magazin ? Daten zur Ökonomie der Freizeit in der Weimarer Republik », in Leiskau, Katja / Rössler, Patrick / Trabert, Susann (dir.), Deutsche illustrierte Presse. Journalismus und visuelle Kultur in der Weimarer Republik, Baden-Baden, Nomos, 25-35.

Dussel, Konrad (2019), Bilder als Botschaft. Bildstrukturen deutscher Illustrierter 1905-1945 im Spannungsfeld von Politik, Wirtschaft und Publikum, Köln, Herbert von Halem.

Feyel, Gilles (2001), " Naissance, constitution progressive et épanouissement d'un genre de presse aux limites floues : le magazine », in Réseaux (105), 19-51.

Frizot, Michel (2009), VU, le magazine photographique, Paris, La Martinière.
Gervais, Thierry / avec la collaboration de Morel, Gaëlle (2015), La fabrique de l'information visuelle : photographies et magazines d'actualité, Paris, Textuel.

Graf, Rüdiger (2008), Die Zukunft der Weimarer Republik. Krisenund Zukunftsaneignungen in Deutschland 1918-1933, München, Oldenbourg

Grevelhörster, Ludger (2005), Der Erste Weltkrieg und das Ende des Kaiserreichs. Geschichte und Wirkung, Münster, Aschendorff.

Jardin, Pierre (2005), Aux racines du mal. 1918. Le déni de la défaite, Paris, Tallandier.

Julien, Élise (2009), Paris, Berlin : la mémoire de la guerre, 1914-1933, Rennes, Presses universitaires de Rennes.

Kalifa, Dominique / Régnier, Philippe / Thérenty, Marie-Ėve / Vaillant, Alain (dir.) (2011), La Civilisation du journal. Histoire culturelle et littéraire de la presse française au XIXe siècle, Paris, Nouveau Monde.

Kott, Christina (2006), Préserver l'art de l'ennemi ? Le patrimoine artistique en Belgique et en France. Bruxelles, Peter Lang.

Krumeich, Gerd (2008), "L'impossible sortie de guerre de l'Allemagne », in Audoin-Rouzeau, Stéphane / Prochasson, Christophe (dir.), Sortir de la Grande Guerre. Le monde et l'après-1918, Paris, Tallandier, 145-164.

Krumeich, Gerd (2018), Die unbewältigte Niederlage. Das Trauma des Ersten Weltkriegs und die Weimarer Republik, Freiburg, Herder. 
Kübler, Hans-Dietrich (2007), « Kriegszeit und demokratischer Umbruch. Die Presse im zweiten Jahrzehnt », in Faulstisch, Werner (dir.), Das zweite Jahrzehnt, München, Fink, 41-71.

Lütgemeier-Davin, Reinhold (1981), « Basismobilisierung gegen den Krieg : Die Nie-wieder-Krieg-Bewegung in der Weimarer Republik ", in Holl, Karl / Wette, Wolfram (dir.), Pazifismus in der Weimarer Republik, Paderborn, Schöningh, 47-76.

Maubach, Franka (2018), « Weimar (nicht) vom Ende her denken », in Aus Politik und Zeitgeschichte, 68 (18-20), 4-9.

Pyta, Wolfram (2007), Hindenburg. Herrschaft zwischen Hohenzollern und Hitler, München, Siedler.

Reimann, Aribert (2004), « Der Erste Weltkrieg - Urkatastrophe oder Katalysator ? ", in Aus Politik und Zeitgeschichte, 54 (2930), 30-38.

Schmitz, Manfred-Guido (2014), Der Erste Weltkrieg und das erste deutsche Massenblatt. Was erfuhr der Leser der Berliner IIIustrirten Zeitung vom politischen Geschehen 1917 und 1918 ?, Nordstrand, Schmitz.

Sloterdijk, Peter (2002), Luftbeben. An der Quelle des Terrors, Frankfurt am Main, Suhrkamp.

Stiftung Deutsches Historisches Museum (dir.) (2017), Die Erfindung der Pressefotografie: aus der Sammlung Ullstein 1894-1945, Berlin, Hantje Cantz.

Thiesse, Anne-Marie (2010), « Rôles de la presse dans la formation des identités nationales », in Thérenty, Marie-Ève / Vaillant, Alain (dir.), Presse, nations et mondialisation au XIX ${ }^{\circledR}$ siècle, Paris, Nouveau Monde, 127-137.

Vowinckel, Annette (2016), Agenten der Bilder. Fotografisches Handeln im 20. Jahrhundert, Göttingen, Wallstein.

Weinrich, Arndt (2017), « Le traumatisme de la défaite. Mémoires et politiques mémorielles en Allemagne - 1918-1939 ", in Jalabert, Laurent / Marcowitz, Reiner / Weinrich, Arndt (dir.), La longue mémoire de la Grande Guerre. Regards croisés franco-allemands de 1918 à nos jours, Villeneuve d'Ascq, Septentrion, 109-122.

Weise, Bernd (1997), « Fotojournalismus. Erster Weltkrieg - Weimarer Republik ", in Honnef, Klaus / Sachsse, Rolf / Thomas, Karin (dir.), Deutsche Fotografie. Macht eines Mediums 18701970, Kunst- und Ausstellungshalle der Bundesrepublik Deutschland, Köln, Dumont, 72-87.

Winter, Jay (2008 [1995]), Entre deuil et mémoire. La Grande Guerre dans l'histoire culturelle de l'Europe, Paris, Colin.

Zunino, Bérénice (2019), Die Mobilmachung der Kinder. Kriegskultur und illustrierte Kinderliteratur im Deutschen Kaiserreich (19111918), Berlin, Peter Lang.

\section{Berliner Illustrirte Zeitung}

« Bei der Entente nach dem Krieg », 26/01/1919, 28 (4), 28.

« Bei der Entente », 09/02/1919, 28 (6), 45.

" Das Schicksal des Feldgrauen. Zur Rückkehr unserer Truppen », 01/12/1918, 27 (48), 378-379.

« Der Raub des Genter Altars », 25/05/1919, 28 (21), 178

« Deutsche Spielwaren », 28/12/1919, 28 (52), 542-543.

« Die Berufskrieger als Kriegsgegner », 19/10/1924, 33 (42), 1218-1219.

« Die Enthüllung des Tannenberg-Denkmals am 10. Jahrestag der Schlacht », 14/09/1924, 33 (37), 1087.
« Die große Kundgebung vor dem Kölner Dom gegen den Vertrag von Versailles am 10. Jahrestag der Unterzeichnung », 14/07/1929, 38 (28), 1226.

« Die Menschen ! Zeitchronik Mai 1919 : Die großartige Leistung des Menschengeistes : das Curtissflugboot überfliegt in 13 Stunden 19 Minuten den Ozean von Amerika nach den Azoren. / In gleicher Zeit verurteilen die Friedensbedingungen der Entente 15 Millionen Deutsche zum Verhungern oder Auswandern », 01/06/1919, 28 (22), 1.

« Die Wirkungen der Hunger-Blockade », 01/06/1919, 28 (22), 192.

« Frau Abgeordnete ! », 09/02/1919, 28 (6), 48.

«Frithjof Nansen - der berühmte norwegische Polarforscher und Staatsmann, der jetzt als Vermittler um Deutschlands Eintritt in den Völkerbund bemüht ist », 19/10/1924, 33 (42), 1.

" Gefallenen-Denkmal in der Kirche in Güstrow (Mecklenburg) von Ernst Barlach », 05/02/1928, 37 (6), 203.

« Kriegsende in Paris und London », 14/12/1918, 27 (50), 394-395.

« Neue Allianzen, neue Kriegsgefahr », 13/01/1924, 33 (2), 22-23.

«Paris nach dem Kriege », 11/05/1919, 28 (19), 154.

« Volk in Not », 21/12/1919, 28 (51), 530-531.

« Weltideen und ihre Apostel », 28/12/1924, 33 (52), 1565-1567.

Boese, Konrad, « Volk in Not. Noch immer ist er in Frankeich kriegsgefangen ! », 21/12/1919, 28 (51), 1.

Brieger, Lothar, «Aus den Frühjahrs-Kunstausstellungen », 08/06/1919, 28 (23), 202-203.

Dyck, Walter von, « Dient die Technik der Kultur? Eine Erwiderung von Walter von Dyck », 15/01/1928, 37 (3), 97-98.

Fahrenkrog, Ludwig, « Das Wiedersehen », 01/12/1918, 27 (48), 1.

Graeser, Erdmann, « Auf der Schattenseite... Bilder aus der Großstadt von Willibald Krain », 14/09/1924, 33 (37), 1085-1087.

«In Weimar vor 10 Jahren », 11/08/1929, 38 (32), 1412-1413.

Kauser, Gustaf, « Die Macht des Zeichners. Ein Rückblick auf die Bild-Propaganda im Kriege », 11/05/1919, 28 (19), 158-159.

Koch-Gotha, Fritz, "Ein Heimatlied auf fremder Erde. (Unsere Kriegsgefangenen dürfen noch immer nicht zurückkehren. Fordern wir stets aufs neue ihre Freigabe !) », 02/02/1919, 28 (5), 35.

Koch-Gotha, Fritz, "Fünfzehn Millionen Deutsche müssten auswandern oder verhungern! Ein Volksredner vor dem Reichstagsgebäude in Berlin bei der großen Demonstration gegen den Erdrosselungsfrieden », 25/05/1919, 28 (21), 1.

Krain, Willibald, « Gefallen an der Somme... Ein Bild zum Totensonntag », 24/11/1929, 38 (47), 2111.

Matejko, Theo, « Ein Zukunftsbild ? Großstadtbild nach einem Gasangriff », 08/09/1929, 38 (36), 1573.

Moszkowski, Alexander, « Dient die Technik der Kultur? », 08/01/1928, 37 (2), 57-59.

Neuburger, Albert, « Was wir im Kriege gelernt haben », 27/04/1919, 28 (17), 130-132.

Oswald, Wilhelm, « Das Schreckgespenst der Welt : Gaskrieg ! Die Selbstvernichtung des Krieges », 08/09/1929, 38 (36), 1572-1574.

« Verfassungsfeier », 26/08/1928, 37 (35), 1460-1461.

Weber, Wolfgang, « Fremdenindustrie auf Schlachtfeldern », 15/07/1928, 37 (29), 1251-1255.

«Zehn Jahre ! Vom 9. November 1918 zum 9. November 1928 ", 11/11/1928, 37 (46), 1956.

«Zehnjahrfeier der Verfassung in Berlin », 25/08/1929, 38 (34), 1490-1491. 